\title{
Microscopic theory of ionic motion in crystals
}

\author{
Aleksandr Rodin ( $\sim$ aleksandr.rodin@yale-nus.edu.sg ) \\ Yale-NUS College https://orcid.org/0000-0001-5321-7690 \\ Keian Noori \\ National University of Singapore \\ Alexandra Carvalho \\ National University of Singapore \\ Antonio Castro Neto \\ National University of Singapore https://orcid.org/0000-0002-1831-322X
}

\section{Physical Sciences - Article}

Keywords: ionic motion, crystals, ionic conduction, solid-state batteries

Posted Date: August 26th, 2021

DOI: https://doi.org/10.21203/rs.3.rs-842228/v1

License: (c) (i) This work is licensed under a Creative Commons Attribution 4.0 International License.

Read Full License 


\section{Microscopic theory of ionic motion in crystals Aleksandr Rodin ${ }^{1,2}$, Keian Noori ${ }^{2}$, Alexandra Carvalho ${ }^{2}$, A. H. Castro Neto ${ }^{2,3}$ \\ ${ }^{1}$ Yale-NUS College, 16 College Avenue West, 138527, Singapore}

${ }^{2}$ Centre for Advanced 2D Materials, National University of Singapore, 117546

${ }^{3}$ Department of Materials Science Engineering, National University of Singapore, 117575

The drive towards an electricity based economy and emerging green technologies has resulted in a tremendous push to create safer and more efficient energy storage devices. ${ }^{1-3}$ The development of solid-state batteries is a major effort in this direction ${ }^{4,5}$. Unlike the case of a traditional electrochemical apparatus, in solid-state batteries ions move through a solid crystalline electrolyte. Ionic motion is thus intimately linked to the condensed matter description of the system - that is, the periodic electronic and ionic properties of the crystal - and is not adequately described by the existing electrochemical tenet. In the present article, we propose a microscopic, first-principles, description of the ionic conduction in crystals. This allows us to understand the ideal characteristics of materials for ionic conduction in general, and for solid-electrolyte applications in particular. Using $a b$ initio calculations, we show that our formalism results in ionic mobilities consistent with experiments for several materials. Our work opens the possibility for the development of solid electrolytes based on fundamental physical principles rather than empirical descriptions of the underlying processes.

The semiconductor technology revolution that occurred in the middle of the $20^{\text {th }}$ century has its origin in the development of the microscopic theory of electron motion in crystals in the early days of quantum mechanics. Trailblazers such as F. Bloch showed the importance of taking into account the periodicity of the crystal structure in order to understand electronic behaviour and the properties of many different types of materials, from metals to semiconductors ${ }^{6}$. Not only did this theoretical framework become important for the understanding of naturally occurring materials, it also allowed for the development of new materials with tailored properties that did not exist before. Electronics turned from a heuristic discipline, based on trial and error, into a predictive science and technology. With the development of superior semiconductor-based technologies, such as the one based on complementary metal-oxide-semiconductors (CMOS), these basic concepts became a common language between scientists and engineers. Such extraordinary developments were driven by the necessity of replacing the obsolete vacuum tube technology that was ubiquitous to the electronic devices of that era.

Arguably, we find ourselves in a similar situation in the area of energy storage. The electrochemical science that has been the basis of battery technology for the last 200 years is faced with the necessity of reinventing itself in order to fulfil the societal needs of today, namely, safe, efficient, reliable, fast, and long-lasting energy storage devices that can be seamlessly incorporated into a modern environmentally conscious lifestyle. The pressure felt by important industrial sectors, such as the automotive, has inspired scientists and engineers to look for alternative solutions to traditional approaches. As such, the field of solid-state batteries has emerged as a possible solution to the conundrum of developing commercially viable electric vehicles ${ }^{4,5,7}$. 
In a solid-state battery, the movable ions, such as lithium $\left(\mathrm{Li}^{+}\right)$, traverse a crystal, that is, a periodic structure consisting of fixed ions (that we call the framework) along with their electrons, and interact with these elements via strong Coulomb forces. Unlike the traditional electrochemical problem in a liquid medium, the ionic motion in crystals depends strongly on their periodicity and symmetries, as in the case of Bloch's theorem. The problem at hand is akin to the famous many-body problem found in strongly interacting electron materials (where phenomena such as magnetism and superconductivity occur) with some fundamental differences, namely, the mass of an ion is approximately 10,000 times that of an electron and while electrons have a strong wave-like characteristics, ions are essentially particles with atomic size. The intricate dance between particles and waves in a crystalline environment is what determines the ionic conductivity and the ultimate efficiency of a solid electrolyte in a battery.

The objective of this work is to develop the basic principles and microscopic formalism that describe ionic motion in crystalline electrolytes. Instead of looking at the problem from a traditional electrochemical perspective, we take a modern condensed matter approach and include the basic elements (mobile and fixed ions and their electrons, in addition to the periodicity of the crystal) from the very beginning. We obtain the steady state equation for the ion motion in a crystal and show that it obeys a Langevin dynamics, consistent with the fluctuation-dissipation theorem, where the ion mobility is determined by the curvature profile of the potential in the atomic lattice and the low frequency phonons of the framework.

In order to substantiate our results, we make use of state-of-the-art density functional theory (DFT) and $a b$ initio molecular dynamics (AIMD) simulations in order to calculate the ion mobility for some crystalline electrolytes and show that our results are consistent with experimental values in polycrystalline samples.

The microscopic Hamiltonian for the problem is given by:

$$
H=K_{e}+V_{e e}+K_{i}+V_{i i}+V_{e i} \text {, (1) }
$$

where $K$ refers to the kinetic energy and $V$ to the Coulomb potential of interactions for electrons (subscript $e$ ) and ions (subscript $i$ ). Given that the mass of the ions is much larger than that of the electrons, we can consider mobile and framework ions to be static in the time scale of motion of electrons (the Born-Oppenheimer approximation). Furthermore, a solid-state electrolyte is characterized to be an insulator for electrons, which implies that the material has a large band gap in the electronic structure so that electron-hole excitations are not created during the ionic motion. This implies that one can use the adiabatic theorem and assume that the ionic motion only leads to smooth modifications of the electron-ion interaction. Within these two standard approximations one is left with an effective ion-ion interaction, $U(\mathbf{r}, \mathbf{u})$, which depends on the position of the mobile ions, $r$, and the framework ions, $\mathbf{u}$ (mathematical details will be presented elsewhere). 
By definition, framework ions are the ones that remain in their crystal lattice position during the flow of the mobile ions. Hence, at any temperature $T$ below the melting point of the crystal, the framework ions undergo oscillatory motion around their equilibrium positions, as illustrated in Fig. 1.

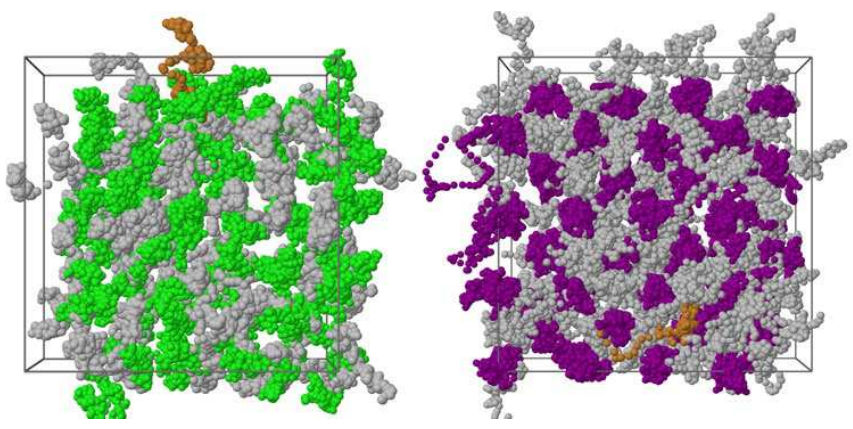

Figure 1: Diffusion trajectories of ions in $\mathrm{AgCl}$ at $600 \mathrm{~K}$ (left panel) and $\alpha$-Agl at $700 \mathrm{~K}$ (right panel), obtained from ab-initio molecular dynamics simulations. Ag atoms are represented in grey, while $\mathrm{Cl}$ and I atoms are represented in green and purple, respectively. The trajectory of a single Ag ion is highlighted in orange. The positions are represented every $0.1 \mathrm{ps,}$ for a total time of $10 \mathrm{ps}$.

Once again, we can use standard solid-state language to describe the framework ions in terms of their phonons, which are characterised by their frequencies $\Omega_{s}$, where the subscript $s$ labels the phonon modes. Of particular importance are the acoustic phonons, or sound modes, with speed of propagation $v_{L, T}$ (where $L$ and $T$ labels the longitudinal and transverse modes). One should stress that these modes do not exist in a liquid or a traditional electrolyte. They only exist in a crystal because a periodicity is induced by the presence of the lattice.

We use the non-equilibrium Keldysh formalism in the path integral representation in order to get the equation of motion for the mobile ions in the form of Newton's equation:

$$
M \ddot{\mathbf{r}}(t)=-\nabla U\left(\mathbf{r}, \mathbf{u}_{0}\right)-\gamma(\mathbf{r}) \dot{\mathbf{r}}(t)+\tilde{\mathbf{f}}(t)+F(\mathbf{r}),(2)
$$

where $t$ is time, $\mathbf{u}_{0}$ is the equilibrium position of the framework ions ( $\nabla$ is the gradient with respect to $r) M$ is the ion mass, $F$ is an applied external force (i.e., electric field),

$$
\gamma(\mathbf{r})=2 \pi \sum_{s} \nabla Y_{S}(\mathbf{r}) \otimes \nabla Y_{S}(\mathbf{r}) \frac{\delta\left(\Omega_{s}\right)}{\hbar \Omega_{S}}
$$

is the dissipative tensor $[\delta(\Omega)$ is the Dirac delta function], where

$$
Y_{S}(\mathbf{r})=\sqrt{\frac{\hbar}{2 \Omega_{S}}}\left[\nabla_{\mathbf{u}} U\left(\mathbf{r}, \mathbf{u}_{0}\right)\right]^{T} \mathbf{m}^{-1 / 2} \varepsilon_{S}
$$

( $\hbar$ is Planck's constant, $\mathbf{m}=\bigoplus m_{j} \mathbf{1}_{D \times D}$ is the framework mass matrix for ions with mass $m_{j}$ and $D$ is the system dimensionality, $\varepsilon_{s}$ is the phonon polarization vector). In Equation (2), the first term on the r.h.s. is a classical term that describes the periodic potential of the static lattice with the framework ions fixed to their equilibrium positions. The second term on the r.h.s. describes the dissipation of energy due to the ion motion when the mobile ion interacts with the phonons of the framework ions. We note that unlike the dynamics in liquids, the dissipative term is position and direction dependent and described in terms of a tensor. Hence, dissipation in a crystal is not isotropic as in a fluid and has quantum nature (as can be seen from the presence of Planck's constant in its definition). This term is rather non-trivial, as we will see below. $\tilde{\mathbf{f}}(\mathbf{r})$ is the fluctuation force due to the vibrations of the framework. Its origin is the same as the dissipative term. One can show that its correlation function is given by: 


$$
\left\langle\tilde{\mathbf{f}}(t) \otimes \tilde{\mathbf{f}}\left(t^{\prime}\right)\right\rangle=\sum_{S} \nabla Y_{S}[\mathbf{r}(t)] \otimes \nabla Y_{S}\left[\mathbf{r}\left(t^{\prime}\right)\right] \operatorname{coth}\left(\frac{\hbar \Omega_{S}}{K_{B} T}\right) \cos \left[\Omega_{S}\left(t-t^{\prime}\right)\right]
$$

where $K_{B}$ is the Boltzmann constant. At high temperatures, $K_{B} T \gg \hbar \Omega_{S}$, we can readily see that:

$$
\gamma(t)=\frac{1}{T} \int_{-\infty}^{t} d t^{\prime}\left\langle\tilde{\mathbf{f}}(t) \otimes \tilde{\mathbf{f}}\left(t^{\prime}\right)\right\rangle(6)
$$

which is a generalized fluctuation-theorem for ionic motion in crystals. Hence, dissipation and fluctuation are intimately related. Once again, unlike in liquids, the fluctuation forces are position and direction dependent.

Having established the existence of a Langevin dynamics of mobile ions in a crystal lattice we can simplify the problem further by considering the steady-state motion in the presence of a constant applied electric field, $E$. In this case, the acceleration and fluctuation terms vanish, and Eq.(2) can be solved for the ion velocity, $\mathbf{v}(\mathbf{r})=\dot{\mathbf{r}}(t)$ :

$$
\mathbf{v}(\mathbf{r})=\gamma(\mathbf{r})^{-1}[-\nabla U(\mathbf{r})+q E],(7)
$$

where $q$ is the ion charge. We now can use the fact that the lattice potential and the dissipation have the periodicity of the lattice and, hence, can be expanded in a Fourier series in terms of reciprocal lattice vectors, $\mathbf{K}$, in order to get:

$$
\mathbf{v}_{\mathbf{K}}=(2 \pi)^{3 / 2} \sum_{\mathbf{K}} \gamma_{\mathbf{K}-\mathbf{K}^{\prime}}^{-1}\left[-\mathbf{i} \mathbf{K}^{\prime} U_{\mathbf{K}^{\prime}}+q E \delta_{\mathbf{K}^{\prime}, 0}\right],
$$

which can be thought of as the equivalent of Bloch's theorem for ionic motion in a crystal. If we are interested only in the average drift velocity we can take $\mathbf{K} \rightarrow 0$ in the above equation and find:

$$
\mathbf{v}_{\text {drift }}=\mathbf{v}_{\mathbf{K} \rightarrow 0}=\left\langle\gamma^{-1}\right\rangle q E,(9)
$$

where

$$
\left\langle\gamma^{-1}\right\rangle=(2 \pi)^{3 / 2} \sum_{\mathbf{K}} \gamma_{\mathbf{K}}^{-1}
$$

is the lattice-averaged dissipation coefficient. From the above expression we can readily obtain the ion mobility:

$$
\mu=\frac{\mathbf{v}_{\mathrm{drift}}}{E}=q\left\langle\gamma^{-1}\right\rangle,
$$

where $\left\langle\gamma^{-1}\right\rangle$ can be computed from first principles for any crystal lattice.

In order to gain more insight into the problem we can simplify the problem considerably by assuming that in Eq. (5) only the acoustic phonon modes contribute to the problem. In this case one can show that:

$$
\gamma(\mathbf{r})=\frac{1}{12 \pi \rho}\left(\frac{1}{v_{L}^{3}}+\frac{2}{v_{T}^{3}}\right)\left[\mathbf{H}_{\mathbf{r}} U(\mathbf{r})\right]^{2},
$$

where $\rho$ is the mass density of the crystal and $\mathbf{H}_{\mathbf{r}}$ is the Hessian operator. We now can see very clearly the dissipative mechanism of ionic motion in a crystal, namely, the softer the crystal (smaller sound velocity) the more dissipative the ionic motion. Furthermore, the movement of the ion is dissipationless in regions of the potential where the Hessian vanishes, which are the saddle point regions of the periodic potential in the unit cell of the crystal. Furthermore, this expression gives us clues regarding what kinds of crystals would be good electrolytes, namely, hard crystals with smooth potential configurations. 
The variations of the potential energy surface can be quantified by computing $U(r)$ from first principles, which we do, as illustration, for the metal-halide electrolytes $\mathrm{AgCl}, \mathrm{LiCl}, \mathrm{Lil}, \alpha-\mathrm{Agl}$, and $\alpha$-CuBr, as shown in Fig. 2 for $\mathrm{AgCl}$ and $\alpha$-Agl. From here, we can obtain $\mu$, per mobile ion, for each compound via the calculation of $\gamma(\mathbf{r})$, as defined in Eq. (12). These ion mobilities, $\mu_{\text {calc, }}$ assuming $q=e$, are listed in Table 1 .

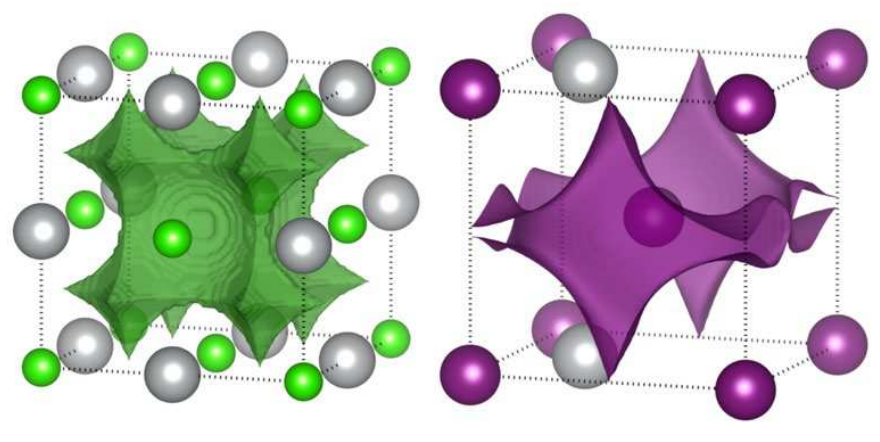

Figure 2: . Three-dimensional potential energy profile, $\mathrm{U}(\boldsymbol{r})$, of a mobile Ag ion for $\mathrm{AgCl}$ (left panel) and $\alpha$-Agl (right panel). The iso-surfaces show the minimum energy at which a continuous connecting pathway exists for the unit cell. The positions of the fixed ions are indicated. Ag atoms are represented in grey, while $\mathrm{Cl}$ and I atoms are represented in green and purple, respectively.

For comparison, we also extract the ionic mobilities per mobile ion, $\mu_{\text {expt, }}$ from available experimental data. The mobility values obtained from fitting the experimental data are lower than the theoretical values, as expected, due to the non-ideal conditions in the experiments. Experimental samples are polycrystalline with reported size and space-charge effects; samples may have more than one mobile defect or ionic species; and the conductivity is usually measured using AC measurements, with sample impedance depending on the frequency. The higher theoretical values show that there is still ample room for improvement in the experimental mobility. Despite these many complications, we note that $\mu_{\text {calc, }}$ computed using only Eq. (11) and simple first-principles inputs, shows a remarkable consistency with $\mu_{\text {expt. }}$

\begin{tabular}{|l|l|l|l|l|l|}
\hline compound & $\mathrm{AgCl}$ & $\mathrm{LiCl}$ & Lil & $\alpha-\mathrm{AgI}$ & $\alpha-\mathrm{CuBr}$ \\
\hline$\mu_{\text {calc }}\left(\mathrm{cm}^{2} \mathrm{~V}^{-1} \mathrm{~s}^{-1}\right)$ & 0.7 & 0.3 & 6.4 & 1.1 & 1.2 \\
\hline$\mu_{\operatorname{expt}}\left(\mathrm{cm}^{2} \mathrm{~V}^{-1} \mathrm{~s}^{-1}\right)$ & 0.08 & - & 0.13 & 0.0022 & $\sim 10^{-3}$ \\
\hline Expt. $\mathrm{E}_{\mathrm{a}}(\mathrm{eV})$ & 0.71 & - & 0.42 & 0.054 & - \\
\hline
\end{tabular}

Table 1: Calculated ionic mobilities per mobile ion $\left(\mu_{\text {calc }}\right)$, together with the values obtained from fitting previous experimental reports $\left(\mu_{\text {expt }}\right)$. The calculated values are directionally-averaged ionic mobilities computed via $v(E q$. (12)), using the potential from ab-initio calculations. The experimental values for the ion mobility and for the activation energy for creation of a mobile ion were obtained from fitting experimental data (see Methods section for details) - AgCl: from Ref. ${ }^{8}$; Lil: from Ref. ${ }^{9} ; \alpha$-Agl: from Ref. ${ }^{10} ; \alpha$-CuBr: from Ref. ${ }^{11}$.

In summary, we have developed a microscopic theory for ionic motion in crystals and derived the equivalent of Bloch's theorem for ions. We found that the ionic mobility depends essentially on the lattice softness (via the third power of the sound velocity) and the curvature of the atomic potential felt by the ions; namely, hard materials with smooth atomic potentials are the best candidates for high ionic mobility. Our calculations for the ionic mobility of several ionic conductors using $a b$ initio methods provide an upper bound for the mobility in single crystals and indicate that the currently measured ionic motilities found in the literature are dominated by extrinsic effects such as impurities, 
grain boundaries, and other types of defects that are already well-known in solid-state physics. Although in the last century we have developed a powerful theoretical framework to study the effect of defects and interfaces in the motion of electrons in solids, the same cannot be said for the case of ions. The understanding of how ions interact with defects and interfaces in solids is an unexplored land. Any further progress in the development of solid-state electrolytes, which are the key elements of solid-state batteries, depends fundamentally on progress in this area of research.

1. [IEA] - International Energy Agency. Global EV Outlook 2020 - Analysis - IEA. lea (2020). Available at: https://www.iea.org/reports/global-ev-outlook-2020. (Accessed: 24th August 2021)

2. Yang, Y., Bremner, S., Menictas, C. \& Kay, M. Battery energy storage system size determination in renewable energy systems: A review. Renew. Sustain. Energy Rev. 91, 109125 (2018).

3. Wang, Q., Mao, B., Stoliarov, S. I. \& Sun, J. A review of lithium ion battery failure mechanisms and fire prevention strategies. Prog. Energy Combust. Sci. 73, 95-131 (2019).

4. Bachman, J. C. et al. Inorganic Solid-State Electrolytes for Lithium Batteries: Mechanisms and Properties Governing Ion Conduction. Chem. Rev. 116, 140-162 (2016).

5. Manthiram, A., Yu, X. \& Wang, S. Lithium battery chemistries enabled by solid-state electrolytes. Nat. Rev. Mats. 2, 1-16 (2017).

6. Hoddeson, L., Baym, G. \& Eckert, M. The development of the quantum-mechanical electron theory of metals: 1928-1933. Rev. Mod. Phys 59, 287-327 (1987).

7. Famprikis, T., Canepa, P., Dawson, J. A., Islam, M. S. \& Masquelier, C. Fundamentals of inorganic solid-state electrolytes for batteries. Nat. Mater. 18, 1278-1291 (2019).

8. Maier, J., Prill, S. \& Reichert, B. Space Charge Effects in Polycrystalline, Micropolycrystalline and Thin Film Samples: Application to AgCl and AgBr. Solid State lonics 1465-1469 (1988).

9. Poulsen, F. Ionic Conductivity of Solid Lithium lodide and its Monohydrate. Solid State lonics 53-57 (1980).

10. Sunandana, C. S. \& Kumar, P. S. Theoretical approaches to superionic conductivity. Bull. Mater. Sci. 27, 1-17 (2004).

11. Funke, K. Solid State Ionics: From Michael Faraday to green energy - The European dimension. Sci. Technol. Adv. Mater. 14, 43502-50 (2013).

12. Giannozzi, P. et al. QUANTUM ESPRESSO: A modular and open-source software project for quantum simulations of materials. J. Phys. Condens. Matter 21, 395502 (2009).

13. Giannozzi, P. et al. Advanced capabilities for materials modelling with Quantum ESPRESSO. J. Phys. Condens. Matter 29, 465901 (2017).

14. Blochl, P. E. Projector augmented-wave method. Phys. Rev. B 50, 17953 (1994).

15. Dal Corso, A. Pseudopotentials periodic table: From H to Pu. Comput. Mater. Sci. 95, 337-350 (2014).

16. Perdew, J. P., Burke, K. \& Ernzerhof, M. Generalized Gradient Approximation Made Simple. Phys. Rev. B 77, 3865 (1996).

17. Wood, B. C. \& Marzari, N. Dynamical structure, bonding, and thermodynamics of the superionic sublattice in $\alpha$-Agl. Phys. Rev. Lett. 97, 166401 (2006). 
18. Hamann, D. R. Optimized norm-conserving Vanderbilt pseudopotentials. Phys. Rev. B 88, 085117 (2013).

19. Schlipf, M. \& Gygi, F. Optimization algorithm for the generation of ONCV pseudopotentials. Comput. Phys. Commun. 196, 36-44 (2015).

20. Setyawan, W. \& Curtarolo, S. High-throughput electronic band structure calculations: Challenges and tools. Comput. Mater. Sci. 49, 299-312 (2010).

21. Momma, K. \& Izumi, F. VESTA 3 for three-dimensional visualization of crystal, volumetric and morphology data. J. Appl. Crystallogr. 44, 1272-1276 (2011).

22. Soler, J. M. et al. The SIESTA method for ab initio order-N materials simulation. J. Phys. Condens. Matter 14, 2745-2779 (2002).

23. Ceperley, D. M. \& Alder, B. J. Ground state of the electron gas by a stochastic method. Phys. Rev. Lett. 45, 566-569 (1980).

24. Troullier, N. \& Martins, J. L. Efficient pseudopotentials for plane-wave calculations. Phys. Rev. B 43, 1993-2006 (1991).

25. Sánchez-Portal, D., Ordejón, P., Artacho, E. \& Soler, J. M. Density-functional method for very large systems with LCAO basis sets. International Journal of Quantum Chemistry 65, 453-461 (1997).

26. Sánchez-Portal, D., Junquera, J., Paz, Ó. \& Artacho, E. Numerical atomic orbitals for linearscaling calculations. Phys. Rev. B 64, 235111 (2001).

27. Nosé, S. A unified formulation of the constant temperature molecular dynamics methods. J. Chem. Phys. 81, 511-519 (1984). 


\section{Methods}

\section{Density functional theory simulations at $0 \mathrm{~K}$}

DFT calculations are performed using the Quantum ESPRESSO ${ }^{12,13}$ code. Structural relaxations and total energy calculations are performed using a PAW basis ${ }^{14,15}$ and the Perdew-Burke-Ernzerhof (PBE) exchange-correlation functional ${ }^{16}$. The kinetic energy cutoffs of the charge density and wavefunctions are set to at least the minimum recommended values of the PAW pseudopotential ${ }^{15}$. The Brillouin zones for all materials are sampled using a uniform $6 \times 6 \times 6$ grid of $K$-points.

For the calculation of $U(\mathbf{r})$ we allow one ion of the mobile metal species to move while keeping all other ions fixed. The mobile ion is moved within the cubic unit cell by intervals of 1/64 of the lattice parameter, $a$. Only configurations in which the distance from the mobile ion to any fixed ion is greater than (5/12)a ( $\alpha-\mathrm{Agl}$ and $\alpha-\mathrm{CuBr}$ ) or (1/3)a (AgCl, LiCl, Lil) are permitted. In the cases of $\alpha-\mathrm{AgI}$ and $\alpha-$ $\mathrm{CuBr}$ we note that the metal ions have partial occupation and thus multiple possible positions. We therefore compute the total energies of all possible permutations of the positions of the mobile ions within the unit cell. The resulting minimum energy configurations are those in which the metal ions are located at the tetrahedral positions on adjacent faces, in agreement with AIMD calculations for $\alpha$ -Agl ${ }^{17}$. Accordingly, to compute $U(\mathbf{r})$ for these materials we fix one of the tetrahedral metal ions and allow the other to move to all other permitted positions, as described above.

Phonon calculations are performed using SG15 optimized norm-conserving Vanderbilt (ONCV) pseudopotentials ${ }^{18,19}$ and a PBE exchange-correlation functional, with a 60 Ry kinetic energy cutoff for wavefunctions. Sound velocities are derived from the phonon dispersions along the $\Gamma-X$ path for cubic cells, as defined in Ref. ${ }^{20}$.

The volumetric images shown in Fig. 2 were generated in VESTA. ${ }^{21}$

\section{Ab initio molecular dynamics simulations}

AIMD simulations are carried out using the SIESTA code. ${ }^{22}$

The forces are calculated using the local density approximation (LDA) of density functional theory, ${ }^{23}$ and a Harris functional is used for the first step of the self-consistency cycle.

The core electrons are represented by pseudopotentials of the Troullier-Martins scheme. ${ }^{24}$

The basis sets for the Kohn-Sham states are linear combinations of numerical atomic orbitals, of the polarized double-zeta type..$^{25,26}$

The $\Gamma$-point is used for Brillouin zone sampling.

a-AgI AIMD calculations were are performed in 256 atom supercells. AIMD calculations for rocksalt structures are performed in 216 atom supercells.

The temperature is controlled by means of a Nosé thermostat ${ }^{27}$. The integration time step used is $1 \mathrm{fs}$ and the total integration time is $26 \mathrm{ps}$. The equilibration time varies between different temperatures and systems and is determined by examining the mean square displacement.

\section{Calculations of the ionic mobility from existing experimental data}

Experimental mobilities are found by fitting existing experimental data in the literature. We have assumed that there is only one mobile ion species. The conductivity is fitted using $\sigma=q n \mu$, where $n=$ $N \exp \left(-E_{\mathrm{a}} / k T\right)$ is the number of mobile ions. Here, $N$ is the total number of atoms of the mobile species in the crystal, $E_{\mathrm{a}}$ is the activation energy necessary to make the ion mobile, $k$ is the Boltzmann constant and $T$ is the temperature. 
Acknowledgements We acknowledge the National Research Foundation, Prime Minister Office, Singapore, under its Medium Sized Centre Programme. A.R. thanks the support by Yale-NUS College (through Grant No. R-607-265- 380-121). The computational work was supported by the Centre of Advanced 2D Materials, funded by the National Research Foundation, Prime Minister's Office, Singapore, under its Medium-Sized Centre Programme.

Author contributions A.R. and A.H.C.N. conceived the research. A.R. performed the theoretical derivations. K.N. performed the DFT calculations and analysis relating to the computation of the ion mobilities. A.C. performed the AIMD simulations and extracted all experimental mobilities. All authors contributed to the writing of the manuscript.

Additional information Correspondence and requests for materials should be addressed to A.R. or A.H.C.N.

Competing interests The authors declare no competing financial interests. 